\title{
GTRACK: A PC program for computing Goldstein's growth constancy index and an alternative measure of tracking
}

\author{
Amy M. Furey ${ }^{a}$, Charles J. Kowalski ${ }^{b}$, Emet D. Schneiderman*c, Stephen M. Willis ${ }^{\mathrm{c}}$ \\ ${ }^{a}$ Department of Biostatistics, 'bepartment of Biologic and Materials Sciences, University of Michigan, Anm Arbor, MI 48109, USA \\ ${ }^{\mathrm{c} D e p a r t m e n t}$ of Oral and Maxillofacial Surgery, Baylor College of Dentistry, 3302 Gaston Ave., Dallas, TX 75246, USA
}

(Received 16 September 1993; accepted 15 October 1993)

\begin{abstract}
This paper reviews Goldstein's 'growth constancy index,' $\xi$, a measure of tracking which can be used to determine whether or not individuals maintain their relative positions in the distribution of a given measurement as that distribution changes over time. We suggest that $\xi$ is an appropriate measure of tracking when the (standardized) measurements arise in the context of a Model I ANOVA, but that the intraclass correlation coefficient, $r_{l}$, may be preferred when a Model II ANOVA is applicable. We also describe - and make available - a PC program which allows the user to choose between Model I and Model II, and computes the appropriate tracking index and confidence intervals for the corresponding parameter.
\end{abstract}

Keywords: Longitudinal studies; Tracking; Repeated measurements; Growth stability

\section{Introduction}

Goldstein's [1] growth constancy index is a measure of tracking, the tendency of individuals to maintain their relative positions in a response distribution as that distribution changes over time. A number of approaches to the quantification of this phenomenon have been taken [2-4] and a good overview of these is available [5]. In addition, programs implementing several of these methods have been written and made available to the biomedical

* Corresponding author. research community [6-10]. The purposes of the present paper are to describe Goldstein's index, which we call $\xi$; to note that it is equal to a perhaps more familiar quantity, $\eta^{2}$, the correlation ratio; to suggest that while $\xi$ is appropriate in the context of a Model I ANOVA, the intraclass correlation coefficient, $r_{l}$, may be preferred in the more frequently encountered (in the context of tracking) case of a Model II ANOVA; and to describe and illustrate a stand-alone, menu-driven $P C$ program, written in GAUSS [11], which can be used to compute either or both of these quantities and confidence intervals for the corresponding parameters. We begin by outlining the development of $\xi$. 


\section{Goldstein's growth constancy index}

We consider one-sample longitudinal data sets of the form

$\mathbf{X}=\left[\begin{array}{cccc}x_{11} & x_{12} & \ldots & x_{1 T} \\ x_{21} & x_{22} & \ldots & x_{2 T} \\ \vdots & \vdots & \ldots & \vdots \\ \mathrm{x}_{\mathrm{N} 1} & x_{N 2} & \ldots & x_{N T}\end{array}\right]$

where $x_{i j}$ denotes the value of the measurement on the $i$ th individual $(i=1,2, \ldots, N)$ at time $t_{j}$ $(j=1,2, \ldots, T)$. The times of measurement $t_{1}, t_{2}, \ldots$, $t_{T}$ need not be equally spaced but are assumed to be the same for each of the $N$ individuals. The first step in computing the growth constancy index is to replace the data matrix $X$ by $Z$ where $Z$ contains the standardized scores for each individual at each time of measurement. That is,

$z_{i j}=\frac{x_{i j}-\bar{x}_{\cdot j}}{s_{j}}$

where $\overline{\mathrm{x}}_{\mathrm{j}}$ is the mean of the $N$ measurements at time $t_{j}$ and $\mathrm{s}_{j}$ the corresponding standard deviation. To the extent that these values remain constant over time for a given individual, that individual is tracking by maintaining his/her position in the (standardized) response distribution. A measure of the departure from tracking for this individual is

$D_{i}^{2}=\sum_{j=1}^{T}\left(z_{i j}-\bar{z}_{i .}\right)^{2}$

where

$\bar{z}_{i}=\frac{1}{T} \sum_{j=1}^{T} z_{i j}$

is the mean value of the standardized observations for the $i$ th individual over the $T$ time points. For the sample as a whole, $\sum_{i=1}^{N} D_{i}^{2}$ then provides a measure of non-constancy and Goldstein defined his growth constancy index as

$\xi=1-\frac{1}{(N-1) T} \sum_{i=1}^{N} D_{i}^{2}$ describing it as, 'the proportion of the total variation $T(N-1)$ not attributable to individuals' variations about their mean (standardized) measurement.' He also noted that the individual values $\mathrm{D}_{i}{ }^{2}$ are measures of each individual's tracking behavior, allowing the comparison of individuals or groups of individuals.

This index has value 1 when tracking is perfect (i.e., when $\Sigma \mathrm{D}_{i}{ }^{2}=0$ ) but can assume positive values in situations representing no tracking, e.g., when the correlations between the successive $z$ values are all zero [1,3]. Accordingly, Goldstein [1] also considered the modified index

$\xi^{*}=\frac{\xi-1 / T}{1-1 / T}$

which continues to equal unity when tracking is perfect, but has value zero when the correlations between the successive $z$-values are zero. Indeed, $\xi^{*}=\overline{\mathrm{r}}$ where $\overline{\mathrm{r}}$ is the average value of these correlation coefficients [1,3]. He also noted that, at least for certain types of measurements, it might be desirable to recognize that some of the variation in growth may be due to 'measurement error' and to 'disattenuate the intercorrelations by subtracting known or estimated measurement error variances from the between-individual variances.' Note that Equation (6) also shows that $\xi=1 / T$ when the correlations are all zero. Thus $\xi^{*}$ is analogous to the chance-corrected tracking index based on the kappa statistic $[4,7]$ : it represents the amount of tracking possible beyond chance that was realized in the data.

\section{The standard error of $\xi^{*}$}

Goldstein proposed the use of a technique known as the jackknife $[12,13]$ to obtain the standard error of the estimator of his stability index and hence confidence intervals for it. As implemented in our program, the jackknife can be described as follows. Let $\xi^{*}$ be the value of the estimator (6) based on all $N$ observations. Now delete the $i$ th observation and let $\xi^{*}{ }_{-1}$ denote the value of the estimator based on the remaining $N-1$ observations. Define

$\tilde{\xi}_{i}^{*}=N \xi^{*}-(N-1) \xi^{*}-1$ 
for $i=1,2, \ldots, N$. The jackknifed estimator of $\xi^{*}$ is then

$$
\tilde{\xi}^{*}=\frac{1}{N_{i}} \sum_{i=1}^{N} \tilde{\xi}_{i}^{*}
$$

and, for large $N$, an approximate $95 \%$ confidence interval for $\xi^{*}$ is

$$
\tilde{\xi}^{*} \pm 1.96 \sqrt{\frac{1}{N(N-1)} \sum_{i=1}^{N}\left(\tilde{\xi}_{i}^{*}-\tilde{\xi}^{*}\right)^{2}}
$$

If $(a, b)$ is the $95 \%$ confidence interval for $\tilde{\xi}^{*}$ the $95 \%$ confidence interval for $\tilde{\xi}$ is $[a(T-1)+1] / T$ $<\xi<[\mathrm{b}(T-1)+1] / T$. We might note that, in our program, to enhance the accuracy of the jackknife procedure [13, p. 140] we jackknife logit $\left(\xi^{*}\right)=\ln \left[\xi^{*} /\left(1-\xi^{*}\right)\right]$ instead of $\xi^{*}$ itself.

In any event, our program provides the overall estimates $\xi$ and $\xi^{*}$, the jackknifed estimates $\tilde{\xi}$ and $\widetilde{\xi}^{*}$ and $95 \%$ confidence intervals for both $\xi$ and $\xi^{*}$. The jackknifed estimators not only allow us to compute the corresponding confidence intervals; they also reduce any bias that $\xi$ and $\xi^{*}$ may have [1]. We also print the occasion-to-occasion correlation matrix, $\mathbf{R}$. While we know $\xi^{*}=\bar{r}$, inspection of $\mathbf{R}$ may reveal patterns among the correlations which can be exploited in further analysis [14, p. 225].

This, then, is a description of Goldstein's growth constancy index. We next relate it to oneway ANOVA in order to show the circumstances in which it may best be used and to offer an alternative which may be more useful in certain situations.

\section{Formulation of $\xi$ in terms of ANOVA}

While Goldstein did implicitly suggest that $\xi$ could be related to ANOVA by noting that it had an interpretation in terms of the proportion of the total variability not due to departures from tracking, he did not pursue this tack. In order to complete the characterization of $\xi$ in terms of the ANOVA, we view the data matrix $\mathrm{Z}$ as consisting of $N$ groups of observations, $T$ observations per group. The measurements $z_{i j}$ are assumed to have the structure

$z_{i j}=\tau_{i}+\epsilon_{i j}$

where $\tau_{i}$ may be viewed as the steady-state or 'true' value for the $i$ th individual $[14$, p. 2], and the $\epsilon_{i j}$ values are random quantities, usually described simply as 'measurement error.' In both Model I and Model II ANOVA we assume $\epsilon_{i j} \sim N\left(0, \sigma_{\epsilon}{ }^{2}\right)$. The difference arises in how the $\tau_{i}$ are structured and interpreted.

In Model I (or fixed-effects ANOVA), the $\tau_{i}$ are considered to be (unknown) constants; in Model II (or random-effects ANOVA) they are considered to be random variables. In the latter situation we assume the $\tau_{i}$ are normally distributed, independently of the $\epsilon_{i j}$, with variance $\sigma_{\tau}^{2}$. The general form of the ANOVA is much the same in Models I and II: the partioning of the sums of squares (SS) is the same, SST $=\mathrm{SSB}+\mathrm{SSW}$; the corresponding numbers of degrees of freedom are the same; and in both cases the expected value of MSW is $\sigma_{\epsilon}{ }^{2}$. The major difference is in MSB which in Model I estimates $\sigma_{\epsilon}{ }^{2}+T \Sigma \tau_{i}{ }^{2} /(N-1)$; but in Model II estimates $\sigma_{\boldsymbol{\epsilon}}{ }^{2}+T \sigma_{\tau}{ }^{2}$. One consequence of this difference is that in Model $I$, inferences are limited to the $N$ cases (individuals) actually included in the study; in Model II, inferences apply to the population from which individuals represent a random sample. Another concerns the way in which one estimates the proportion of the total variability due to individual differences $[15$, p. 382]. The concept remains the same, but different methods of estimation are employed. In Model I, an appropriate measure [15, p. 349] of the extent to which 'treatments' (in our case, individuals) are accounting for variance in the dependent variable is $\eta^{2}=\mathrm{SSB} / \mathrm{SST}$, which is sometimes referred to as the 'correlation ratio.' It is easy to see that this is the same as $\xi$. Simply recognize $\Sigma D_{i}^{2}$ as SSW and since SST $=\mathrm{SSB}+$ $\mathrm{SSW}=T(N-1)$,

$\xi=\frac{T(N-1)-\sum D_{i}^{2}}{T(N-1)}=\frac{\text { SSB }}{\text { SST }}$

Thus $\xi$ is an appropriate measure in the case of Model I. 
In the case of Model II ANOVA, where the individuals are viewed as a random sample, and the variance of an observation $z_{i j}$ is $\mathrm{V}\left(z_{i j}\right)=\sigma_{\tau}{ }^{2}+\sigma_{\epsilon}{ }^{2}$, the appropriate measure of variance accounted for is the intraclass correlation coefficient, $r_{I}$, defined by

$r_{I}=\frac{\sigma_{\tau}^{2}}{\sigma_{\tau}^{2}+\sigma_{\epsilon}^{2}}$

i.e., the proportion of variance in an observation due to subject-to-subject variability in their steady-state scores. This is estimated by $[14$, p. 11 ; 15 , p. $382 ; 16$, p. 126$]$

$\hat{r}_{\mathrm{I}}=\frac{\mathrm{MSB}-\mathrm{MSW}}{\mathrm{MSB}+(T-1) \mathrm{MSW}}$

which is somewhat different than [11]. The relationship between the two may be seen from

$$
\begin{aligned}
\xi=\frac{\mathrm{SSB}}{\mathrm{SST}} & =\frac{\mathrm{SSB}}{\mathrm{SSB}+\mathrm{SSW}} \\
& =\frac{(N-1) \mathrm{MSB}}{(N-1) \mathrm{MSB}+N(T-1) \mathrm{MSW}} \\
& \approx \frac{\mathrm{MSB}}{\mathrm{MSB}+(T-1) \mathrm{MSW}}
\end{aligned}
$$

i.e., for large $N, \xi$ is essentially the same as the intraclass correlation except that MSW has not been subtracted from MSB in the numerator. Since MSW estimates $\sigma_{\epsilon}{ }^{2}$, this is presumably the sense in which Goldstein noted that $\xi$ 'has not been corrected' for measurement error: Goldstein's 'disattenuated' version of $\xi$ is simply the intraclass correlation coefficient. Note that $\xi \geq \hat{r}_{\text {I }}$, i.e., that if $\xi$ is used when in fact Model II is appropriate, $\xi$ will overestimate stability. This is not surprising since measurement error inflates SSB (recall MSB estimates $T \sigma_{\tau}{ }^{2}+\sigma_{\epsilon}{ }^{2}$ ).

In any event, we should note that it is not necessary to 'correct' $\hat{r}_{\mathrm{i}}$ as in Eq. (6): when $\bar{r}=0, \hat{r}_{\mathrm{i}}=0[16, \mathrm{p} .126]$. The statistic $\hat{r}_{\mathrm{i}}$ also has an interpretation in terms of the average occasionto-occasion correlation coefficients: $\hat{r}_{\mathrm{i}}=\bar{r}$ when the pooled estimate of variance is used in the denominator of all the correlations [16, p. 126].

\section{Confidence intervals for $\mathbf{r}_{\mathbf{l}}$}

In constructing confidence intervals for $\xi$ and/or $\xi^{*}$, jackknifing was used to estimate the corresponding standard error. An advantage of using the intraclass correlation coefficient (when appropriate) is that a closed form expression for confidence intervals exists. If the $\tau_{i}$ and $\epsilon_{i j}$ are normally distributed, $(1-\alpha) \times 100 \%$ confidence intervals for $r_{I}$ can be constructed from $[15$, p. $383 ; 17$, p. 652$]$

$\frac{\mathrm{L}}{\mathrm{L}+1} \leq r_{I} \leq \frac{\mathrm{U}}{\mathrm{U}+1}$

where

$$
\begin{aligned}
\mathrm{L}= & \frac{1}{T}\left[\frac{\mathrm{MSB}}{\mathrm{MSW}}\right. \\
& \left.\times \frac{1}{\mathrm{~F}(1-\alpha / 2 ; N-1, N(T-1))}-1\right]
\end{aligned}
$$

and

$$
\mathrm{U}=\frac{1}{T}\left[\frac{\mathrm{MSB}}{\mathrm{MSW}} \times \frac{1}{\mathrm{~F}(\alpha / 2 ; N-1, N(T-1))}-1\right]
$$

Some investigators might prefer to use the onesided version of this confidence interval: the onesided $(1-\alpha) \times 100 \%$ confidence interval for $r_{I}$ is given by $[14$, p. 12]:

$$
r_{I} \geq \frac{\frac{\mathrm{MSB}}{\mathrm{MSW}}-\mathrm{F}(1-\alpha ; N-1, N(T-1))}{\frac{\mathrm{MSB}}{\mathrm{MSW}}+(T-1) \mathrm{F}(1-\alpha ; N-1, N(T-1))}
$$

In the above, $F(\alpha, d f 1, d f 2)$ denotes the $\alpha$ th percentile of the F-distribution with $\mathrm{df} 1$ and $\mathrm{df} 2$ degrees of freedom. To compute values not generally tabulated, the reader may use the relationship $F_{\alpha}(d f 1, d f 2)=1 / F_{1-\alpha}(d f 2, d f 1)$. For example, $F_{.025}(11,48)=1 / F_{.975}(48,11)=0.33$.

\section{An example}

We illustrate the methods and our program using the data consisting of $T=5$ measurements 
of mandibular ramus height in $N=12$ young male rhesus monkeys previously considered in [18] and shown below:

$\begin{array}{llllll}\text { Monkey } & \text { Timel } & \text { Time2 } & \text { Time3 } & \text { Time4 } & \text { Time5 } \\ 1 & 25.2 & 29.0 & 33.6 & 35.2 & 35.8 \\ 2 & 27.3 & 32.1 & 37.0 & 41.8 & 43.5 \\ 3 & 26.3 & 30.7 & 36.1 & 38.0 & 38.9 \\ 4 & 26.0 & 34.5 & 39.0 & 42.3 & 44.4 \\ 5 & 25.5 & 29.5 & 34.4 & 38.3 & 37.9 \\ 6 & 28.2 & 32.5 & 36.3 & 42.3 & 43.8 \\ 7 & 25.4 & 33.4 & 38.0 & 42.7 & 43.1 \\ 8 & 27.2 & 34.8 & 37.2 & 44.0 & 44.0 \\ 9 & 26.0 & 34.5 & 38.0 & 43.5 & 43.8 \\ 10 & 28.5 & 33.8 & 38.0 & 39.2 & 42.0 \\ 11 & 27.0 & 31.2 & 36.0 & 41.7 & 43.8 \\ 12 & 26.0 & 33.0 & 40.2 & 42.5 & 43.8\end{array}$

The program is invoked by the command

GSRUNI GTRACK. asked (1) what is the name of the ASCII file containing the data (the file name must have the ASC extension); (2) what is the number of time points, $T$. The program determines the number of subjects, $N$; The user is then asked to choose between MODEL I (fixed-effects ANOVA) and MODEL II (random-effects ANOVA). If I, Goldstein's indices are computed and confidence intervals for them are produced by jackknifing. If II, the intraclass correlation is computed and confidence intervals constructed according to [14] and [15].

The output then appears on the screen and is simultaneously written to a output file on disk in ASCII format named GTRACK.OUT. This file may be annotated and printed using most word processors or the DOS [PRN] utility.

For the example data [18], where we view the 12 monkeys as comprising a sample from some larger population and hence choose Model II, the output is as follows. The $\mathrm{Z}$ matrix, the individual standardized means, and the $D^{2}$ values are given by

\begin{tabular}{crrrrrrr} 
Monkey & \multicolumn{1}{c}{$\mathrm{Z1}$} & \multicolumn{1}{c}{$\mathrm{Z} 2$} & \multicolumn{1}{c}{$\mathrm{Z3}$} & \multicolumn{1}{c}{$\mathrm{Z} 4$} & \multicolumn{1}{c}{$\mathrm{Z} 5$} & \multicolumn{1}{c}{$\mathrm{Z}$} & $\mathrm{D}^{2}$ \\
1 & -1.245 & -1.742 & -1.823 & -2.162 & -2.179 & -1.830 & 0.582 \\
2 & 0.692 & -0.161 & 0.009 & 0.316 & 0.498 & 0.271 & 0.486 \\
3 & -0.231 & -0.8755 & -0.476 & -1.111 & -1.101 & -0.759 & 0.614 \\
4 & -0.507 & 1.062 & 1.087 & 0.504 & 0.811 & 0.591 & 1.730 \\
5 & -0.968 & -1.487 & -1.392 & -0.998 & -1.449 & -1.259 & 0.258 \\
6 & 1.522 & 0.042 & -0.368 & 0.504 & 0.603 & 0.461 & 2.010 \\
7 & -1.061 & 0.501 & 0.548 & 0.654 & 0.359 & 0.200 & 2.033 \\
8 & 0.600 & 1.215 & 0.117 & 1.142 & 0.672 & 0.749 & 0.800 \\
9 & -0.507 & 1.062 & 0.548 & 0.954 & 0.603 & 0.532 & 1.545 \\
10 & 1.799 & 0.705 & 0.548 & -0.660 & -0.023 & 0.474 & 3.347 \\
11 & 0.415 & -0.620 & -0.530 & 0.278 & 0.603 & 0.029 & 1.274 \\
12 & -0.507 & 0.297 & 1.733 & 0.579 & 0.603 & 0.541 & 2.584
\end{tabular}

The user is then prompted for some basic information regarding the longitudinat data set to be analyzed and how it is to be analyzed. The user is
The user is given the opportunity to save the $D^{2}$ values in an ASCII file for further analysis. Note that, in this example, these values readily 
distinguish those individuals who are tracking from those who are not. The usual ANOVA table is then printed

$\begin{array}{lccc}\text { Source } & \text { df } & \text { SS } & \text { MS } \\ \text { Between } & 11 & 37.737 & 3.431 \\ \text { Within } & 48 & 17.263 & 0.360 \\ \text { Total } & 59 & 55 & \end{array}$

From this we compute $\bar{r}_{1}=0.631$ and the twosided $95 \%$ confidence interval is

$$
0.390 \leq r_{I} \leq 0.848 \text {. }
$$

The corresponding one-sided $95 \%$ confidence interval for the intraclass correlation coefficient is $r_{I} \geq 0.431$. We also print the $T \times T$ inter-period correlation matrix

$\begin{array}{llllll}\text { Time } & \text { T1 } & \text { T2 } & \text { T3 } & \text { T4 } & \text { T5 } \\ \text { T1 } & 1 & & & & \\ \text { T2 } & 0.340 & 1 & & & \\ \text { T3 } & 0.156 & 0.821 & 1 & & \\ \text { T4 } & 0.253 & 0.817 & 0.703 & 1 & \\ \text { T5 } & 0.440 & 0.832 & 0.772 & 0.942 & 1\end{array}$

\section{Discussion}

The intraclass correlation coefficient, the appropriate index for use when the monkeys are considered to represent a random sample, has value $\hat{r}_{l}=0.631$. If we would have used instead the analogous form (the one with an interpretation as the proportion of variance accounted for) of Goldstein's stability index we would obtain $\xi=0.686$. $\xi$, as noted earlier, overestimates stability. On the other hand, the corrected form of Goldstein's index (6) has the value $\xi^{*}=0.608$. It may also be noted that $\xi^{*}=\bar{r}$, the average of the correlation coefficients in the above table. The jackknifed estimators and corresponding $95 \%$ confidence intervals are, respectively, $0.705(0.442$, $0.897)$ and $0.631(0.303,0.871)$.

It may be of interest to compare these values with those of other tracking indices. For the index based on Cohen's kappa statistic using 3 tracks
[7], $\kappa=0.242$ with $95 \%$ confidence interval $(0.113$, 0.372 ). For the unstructured form of the FoulkesDavis tracking index [2,8], also considered in [1], the value is FDI $=0.394$ and the corresponding confidence interval is $(0.245,0.543)$. For the more structured form of this index, where polynomials are fit before the number of crossings are counted ( $\mathrm{D}=2$ is satisfactory at $\alpha=0.05$ ), FDII $=0.530$ and $(0.400,0.660)$. While these indices are not in 'good agreement', differences between them may be expected since they measure different aspects of 'tracking behavior' $[1,6]$. In the case of the kappa index, the value before chance-correction is 0.500 , which is more in line with the values of the other indices.

Goldstein also compared his stability index to FDI and FDII. He computed these indices for height, weight and skinfold measurements and found:

$\begin{array}{lllll} & \xi & \text { FDI } & \begin{array}{l}\text { FDII } \\ (\mathrm{D}=1)\end{array} & \begin{array}{l}\text { FDII } \\ (\mathrm{D}=2)\end{array} \\ \text { Height } & 0.97 & 0.85 & 0.88 & 0.86 \\ \text { Weight } & 0.95 & 0.82 & 0.86 & 0.85 \\ \text { Skinfold } & 0.75 & 0.58 & 0.70 & 0.64\end{array}$

It is seen that the values of the indices considered can vary markedly and depend on such factors as the nature of the measurement considered, the number of tracks for kappa [6,7], the time interval over which the measurements are taken, and the degree of the polynomial fit to smooth the data.

In the context of the indices (5), (6) and (12), the value will also depend on whether Model $I$ or Model II is selected by the user. While we suspect that in most applications of our program Model II will be appropriate (and hence $r_{I}$ will be computed), there are situations in which Model $I$ is appropriate and $\xi$ and/or $\xi^{*}$ should be computed. As an example, consider a university official in the state of Michigan who is interested in seeing whether a defined set of state-supported schools track with respect to their annual appropriations. Since this official is interested only in these particular schools (and does not intend to generalize to other schools), Model $I$ is appropriate and $\xi$ and/or $\xi^{*}$ should be used. We might note that $\xi\left(=\eta^{2}\right)$ is not the only measure which could have 
been used for the stated purpose in Model I situations. Indeed, perhaps the most widely used is $\omega^{2}$ $[19$, p. 23]. Hays $[15$, p. 290] defines this in general terms as

$\omega^{2}=\frac{\sigma_{Y}^{2}-\sigma_{Y \mid X}^{2}}{\sigma_{Y}^{2}}$

i.e., the proportion of variance in the dependent variable $(Y)$ accounted for by the independent variable $(X)$. He notes $\left[15\right.$, p. 291] that $\omega^{2}$ is closely related to $\eta^{2}$ and $r_{1}$, but that these, 'were developed for and used in somewhat different contexts.' $\eta^{2}$ is shown to be appropriate for Model I [15, p. 349]; $r_{\mathrm{I}}$ in Model II [15, p. 382]. A good description is available in $[20$, p. $89 \mathrm{ff}]$. Other alternatives, including $\eta^{2}$ and $r_{\mathrm{I}}$, are discussed in [21].

Returning to the Model II situation, an important use of $\sqrt{\mathrm{MSW}}$ was noted by Fleiss $[14, \mathrm{p} .11]$. For a single subject, the steady-state score $\tau$ may be considered an unknown parameter. If a single observed measurement, $z$, is available on that individual, an approximate $95 \%$ confidence interval for his/her true value of $\tau$ is $z \pm 2 \sqrt{\mathrm{MSW}}$. If the individual is measured $T$ times, and has mean $\bar{z}$, an approximate $95 \%$ confidence interval for $\tau$ is

$$
\bar{z} \pm 2 \sqrt{\frac{\mathrm{MSW}}{T}}
$$

Consider, e.g., a 'new' monkey, measured at but a single point in time, for which $z=1$. Recalling that MSW $=0.36$, the $95 \%$ confidence interval for this monkey's steady-state value, $\tau$, is $(-0.2,2.2)$. If instead $T=4$ time points had been available, the corresponding interval would have been $(0.4,1.6)$. It is thus seen that the methodology developed above may have application in diagnostic/predictive contexts.

\section{Availability}

A full set of PC programs for longitudinal data analysis, including this program, can be obtained on $5.25^{\prime \prime}$ or $3.5^{\prime \prime}$ diskettes (please request type) by sending \$25 to defray the cost of handling and licensing fees. These programs require a 80386 or 80486 based personal computer (PC) running the MS-DOS operating system (version 5.0 or higher is recommended, although versions as low as 3.3 will suffice). 80386 computers must also be equipped with a 80387 math coprocessor. At least $4 \mathrm{MB}$ of memory is required, and must be available to GAUSS386i, i.e., not in use by memory resident programs such as Windows. EGA or VGA graphic capabilities are required to display the color graphics; VGA or SVGA is suggested to display optimally the graphic results. Runtime modules are supplied with the programs so that no additional software (i.e., compiler or interpreter) is required to run these programs. One can create and edit ASCII data sets for use by these programs using the full screen editor supplied with MS-DOS version 5.0. The programs are written and compiled using GAUSS386i, version 3.0, require no additional installation or modification, and are run with a single command. When requesting the programs, address inquiries to the corresponding author and make checks payable to Baylor College of Dentistry.

\section{Acknowledgement}

This research was supported by grant DE08730 from the National Institute of Dental Research.

\section{References}

1 Goldstein H: Measuring the stability of individual growth patterns, Annals Hum Biol, 8 (1981) 549-557.

2 Foulkes MA and Davis CF: An index of tracking for longitudinal data, Biometrics, 37 (1981) 439-446.

3 McMahan CA: An index of tracking, Biometrics, 37 (1981) 447-455.

4 Ware JH and Wu MC: Tracking: prediction of future values from serial measurements, Biometrics, 37 (1981) 427-437.

5 Ware JH: Tracking, The Encyclopedia of Statistical Sciences, (Eds: S Kotz and NL Johnson), Vol. 9, Wiley, New York, 1988.

6 Kowalski CJ and Schneiderman ED: Tracking: concepts, methods and tools, Hum Evolution, in press.

7 Schneiderman ED, Kowalski CJ and Ten Have TR: A GAUSS program for computing an index of tracking for longitudinal data, Am J Hum Biol, 2 (1990) 475-490. 
8 Schneiderman ED, Kowalski CJ, Ten Have TR and Willis SM: Computation of Foulkes and Davis' nonparametric tracking index using GAUSS, Am J Hum Biol, 4 (1992) 417-420.

9 Dallal GE: TRACK: A FORTRAN program for calculating the Foulkes-Davis tracking index, Comput Biol Med, 19 (1989) 367-371.

10 Schneiderman ED, Willis SM, Kowalski CJ and Ten Have TR: A PC program for comparing tracking indices in several independent groups, Am J Hum Biol, 4 (1992) 399-401.

11 Edlefsen LE and Jones SD: GAUSS, Applied Technical Systems, Kent, Wash., 1985.

12 Miller RG: The jackknife - a review, Biometrika, 61 (1974) 1-17.

13 Mosteller F and Tukey JW: Data Analysis and Regression, Addison-Wesley, New York, 1977.

14 Fleiss JL: The Design and Analysis of Clinical Experiments, Wiley, New York, 1986.
15 Hays WL: Statistics, 3rd Edn, Holt, Rinehart and Winston, New York, 1981.

16 Winer BJ: Statistical Principles in Experimental Design, McGraw-Hill, New York, 1962.

17 Neter J, Wasserman W and Kumer MH: Applied Linear Statistical Models, 2nd Edn, Irwin, Homewood, Ill., 1985.

18 Schneiderman ED and Kowalski CJ: Implementation of Rao's polynomial growth curve model using SAS, $A m J$ Phys Anthro, 67 (1985) 323-333.

19 Brown SR and Melamed LE: Experimental Design and Analysis, Sage, Newbury Park, Calif., 1990.

20 Keppel G: Design and Analysis: A Researcher's Handbook, Prentice-Hall, Englewood Cliffs, N.J., 1973.

21 Howell DC: Statistical Methods for Psychology, 2nd Edn, PWS-Kent, Boston, 1987. 